\title{
Adaptive Numerical Analysis of Wrinkling in Sheet Metal Forming
}

\author{
A. Selman*, T. Meinders** , A.H. van den Boogaard** and J. \\ Huétink** \\ * Netherlands Institute for Metals Research - Rotterdamseweg 137 - 2628 AL Delft - \\ The Netherlands \\ ** University of Twente - Department of Mechanical Engineering - P.O. Box. 217 - \\ 7500 AE Enschede - The Netherlands
}

\begin{abstract}
Hutchinson approach has been successfully used by a number of researchers in thin sheet metal forming processes for wrinkling prediction. However, Hutchinson approach is limited to regions of the sheet that are free of any contact. Therefore, a new wrinkling indicator that can be used in the contact areas is proposed. Discretisation error indicators are also used to present a comprehensive approach to wrinkling prediction analysis.
\end{abstract}

KEY WORDS: Numerical Simulation, Finite Elements, Sheet Metal Forming, Error Estimation, Wrinkling Prediction and Adaptive Mesh Refinement. 


\section{Introduction}

Wrinkling is becoming one of the most troublesome modes of failure in sheet metals during stamping and other forming operations mainly because of the trend towards thinner, high-strength sheet metals [HUT 74, 85], [NEA 89, 90], [AMZ 91].

The methods used in the past to predict wrinkling failures in sheet metals have been mostly empirical and have, unfortunately, proved to be inadequate for predicting observed trends.

In a numerical simulation, wrinkles can be detected by a visual inspection of the deformed mesh, provided that the finite element discretisation is fine enough to allow a proper capture of the wrinkles. This, in general, makes it cumbersome to proceed with the analysis. Rather, it is desirable to proceed with a selective refinement to keep the computational cost low (acceptable). In this context, [BON 92], [ROD 97], and more generally in finite element simulations [SEL 90, 93, 97], adaptive mesh refinement plays an essential role. However, this implies that some kind of wrinkling indicators are used to direct the refinement process [SEL 00, 02], [BON 94], [NOR 97, 98], [BRU 97], [GEL 98].

In this work, the analysis of Hutchinson and Neale [HUT 85], which consists of formulating the problem within the context of plastic bifurcation theory for thin shell elements and its extension by Neale [NEA 89] to account for more general constitutive models, is used. Under a number of assumptions, limitations and simplifications a simple wrinkling criterion with some restrictive applicability, is obtained. Nonetheless, the results are used to locally define a wrinkling risk factor or simply a (contact free) wrinkling indicator, which, in turn, is used to detect the zones (elements) to be refined in a subsequent adaptive mesh refinement process. To complete the analysis, the wavelength number is used to determine the appropriate new mesh size.

Hutchinson analysis is, unfortunately, limited to regions of the sheet that are free of any contact. When contact is taken into account the problem is further complicated. Furthermore, given that numerical simulations of complex sheet metal forming involve large scale models, it is obvious that global wrinkling indicators found in the literature - mostly based on eigen value analysis of the global tangent stiffness matrix - should not be used because of their high computational cost. This is to avoid over-loading the already time consuming deep drawing simulations. Consequently, an indicator based on the local change of curvatures will be considered.

Finally, we note that in wrinkling prediction analyses, the local curvature, amongst other parameters such as the thickness, plays a major role and should, therefore, be properly approximated from the finite element meshes at all stages of the computation. In this context, the incorporation of discretisation errors indicators and adaptive mesh refinement in sheet metal forming processes is doubly important 
in keeping the computation cost low and allowing a proper wrinkling prediction analysis.

The general and comprehensive algorithm for mesh adaptation in sheet metal forming processes comprises the following steps [SEL 00, 02]

1. Generate an initial grid to represent the computational domain and to allow an adequate initial solution

2. Advance the solution for a number of steps

3. Use the error indicator and/or wrinkling indicators to determine whether mesh refinement is necessary. If yes, compute a new mesh distribution and continue - otherwise go to step 2

4. Proceed with the mesh refinement and obtain the field values of the solution on the new grid by direct interpolation from the previous grid

5. If the desired load interval has elapsed stop - otherwise go to step 2

In the following key sections, some details are given on how the discretisation errors indicator and the wrinkling prediction indicators are determined and how these are used to compute a new mesh size in an adaptive mesh refinement process.

\section{Discretisation errors estimation}

In contrast to the energy type of error estimators, that have been recently developed for the simulation of $2 \mathrm{D}$ and $3 \mathrm{D}$ bulk forming processes, the error estimation presented by Bonet [BON 94] is entirely geometrical and is based on the accuracy with which the finite element mesh can describe the continuous exact shape. In addition to Bonet's geometric error estimation, a thickness error which measures the jump between the finite element solution and a solution obtained by some recovery technique to substitute for the exact solution, is also taken into account, as the thickness distribution is of primary important in sheet metal forming, and plays an essential role in wrinkling prediction analysis. The two parts of the discretisation errors estimation are now briefly described.

\subsection{Evaluation of the thickness error and size}

The thickness error is evaluated using the following formula 


$$
e_{h}=\frac{1}{A} \int\left|\frac{t-\bar{t}}{t}\right| d A
$$

where $A$ is the element area, $t$ represents the finite element thickness distribution and $\bar{t}$ a smooth thickness distribution to substitute for the exact thickness solution. In this work, a simple, yet efficient, area weighted nodal averaging technique followed by a linear interpolation over each element is used.

Experience has shown that a quadratic relation between this error measure and the element size gives satisfactory results. Therefore, an element for which the size $L$ and thickness error $e_{h}$ are known, will be assigned the following element thickness size to satisfy a user specified tolerance $\eta_{h}$

$$
L^{h}=L \sqrt{\frac{\eta_{h}}{e_{h}}}
$$

\subsection{Evaluation of the geometric error and size}

Although the (local) tangent sets are constant over each element, as linear triangles are used, the variation of these sets from one element to its neighbours gives rise to a quadratic variation of the geometry that cannot be represented by facet elements.

To quantify the variation of the tangent sets of axes, an area weighted nodal smoothing technique is first used to obtain a smooth nodal distribution of the tangent sets. Then, for each $i$-node, the smooth metric tensor $\bar{g}_{i j}$ is determined as the dot product of the smooth tangent vectors $\bar{g}_{i}$ and $\bar{g}_{j}$. As for the tangent sets, this variable varies linearly over each element.

Given a metric tensor corresponding to the actual mesh and a smooth metric tensor corresponding to a higher order surface, the geometric error, as defined by Bonet [BON 94], is obtained by first evaluating the deviation between the two surfaces. This is expressed simply in the surface plane, by the use of a pseudo Green Lagrange tensor $E$ as

$$
E_{i j}=\frac{1}{2}\left(\bar{g}_{i j}-g_{i j}\right) \quad i, j=1,2
$$

of which the contravariant components are given by 


$$
E^{i j}=g^{i k} g^{j l} E_{k l}
$$

The deformation in the normal direction, $E_{33}$ is obtained by considering volume conservation and is expressed as

$$
E_{33}=\frac{1}{2}\left[\frac{g}{\bar{g}}-1\right]
$$

where $g$ and $\bar{g}$ are the determinants of the metric tensor and smooth metric tensor, respectively.

A norm measure of the error tensor is given by its invariant $\Pi_{E}^{2}$ as

$$
\Pi_{E}^{2}=E: E=E^{m n} E_{m n}+E^{33} E_{33}
$$

Henceforth, the local geometric error is evaluated by an integration of this field element wise as

$$
e_{g}^{2}=\frac{1}{A} \int \Pi_{E}^{2} d A
$$

This error norm is, assuming again a quadratic rate of convergence, is translated into a geometric mesh size as

$$
L^{g}=L \sqrt{\frac{\eta_{g}}{e_{g}}}
$$

with $\eta_{g}$ a user specified tolerance not necessarily equal to $\eta_{h}$.

The new mesh size is determined as the minimum of $L^{h}$ and $L^{g}$. However, a minimum size of 2 to 4 times the thickness is set to limit the minimum element size allowed.

The remeshing is started when the mean or maximum value of the relative change in element size over the whole mesh is larger that some specified tolerances. If that is not the case i.e. the actual mesh needs no refinement, then the solution is 
advanced for a preset number of steps as indicated in the algorithm for mesh adaptation in sheet metal forming processes.

\section{Contact free wrinkling analysis}

The basic theory of plastic buckling and relevant relations for the DonnellMushtari-Vlasov (DMV) shallow shell theory have been developed by Hutchinson [HUT 74]. The application of this theory to sheet wrinkling was first carried out by Hutchinson and Neale [HUT 85] and is used in the present work. However, it should be stresses that a number of assumptions, limitations and simplifications are embedded in Hutchinson analysis. First, we consider a sheet element, which, in the current stage of forming, has attained a doubly curved state with principal radii of curvature and thickness, all assumed to be constant over the region of the sheet being examined for susceptibility to local wrinkling. It is also assumed that the stress state prior to wrinkling to be a uniform membrane state over the local region being examined for wrinkling. Although the analysis can account for any stress state, it is for simplicity, assumed that the principal axes of this uniform membrane stress state coincide with the principal axes of curvature. Simplifications arise from the fact that the anticipated short-wavelength modes are shallow and that they can be analysed using DMV shallow shell theory. Finally, the investigation is limited to regions of the sheet that are free of any contact.

\subsection{Hutchinson analysis}

To determine the critical stress state for buckling that is needed in the definition of the wrinkling indicator, Hutchinson bifurcation functional [HUT 74] is used. The analysis involves substituting the fields used to describe wrinkling into the bifurcation functional and integrating over the local area of the sheet over which the wrinkles develop. Buckling is possible when the associated functional $F$ is nil. To determine the critical stress values for which short wavelength buckling first occurs, the determinant of $F$ with respect to the waveform parameters is minimised and the minimum is set to zero. The values of the waveform parameters so obtained describe the corresponding critical buckling pattern.

It should, however, be noted that for the pre-wrinkling geometry and stress state considered above, wrinkling will in most cases be aligned with one of the principal curvatures (stress) directions and that, in such a case, the analysis simplifies considerably.

Moreover, if we assume incompressibility and that the pre-wrinkling loading history is proportional and that deformation theory of plasticity can be used to characterise the material behaviour, a simple wrinkling criterion can be obtained. 
For a power law hardening relation of the form $\sigma_{e}=K \varepsilon_{e}{ }^{n}$, the critical condition, for wrinkling perpendicular to the first principal direction, reduces to

$$
\sigma_{1}^{c r}=K\left[\frac{1+r}{\sqrt{3(1+2 r)}} \frac{t}{R_{2}} \sqrt{n} \alpha_{1}^{1-1 / n}\right]^{n}
$$

for the stress, and

$$
\lambda_{1}^{c r^{2}}=\frac{2 \sqrt{3} \sqrt{n}}{\frac{1+r}{\sqrt{1+2 r}}-\frac{(1-n) \sqrt{1+2 r)}}{(1+r)} \frac{1}{\alpha_{1}^{2}}}
$$

for the wave number. In the above equations, $R_{2}$ is the radius of curvature in the second principal direction evaluated from the finite element mesh [NAG 72] and $r$ is the average Lankford strain ratio and

$$
\alpha_{1}=\left[1-\frac{2 r \alpha}{(1+r)}+\alpha^{2}\right]^{1 / 2}
$$

in which $\alpha=\sigma_{2} / \sigma_{1}$ is the proportionality factor.

\subsection{Wrinkling indicator}

Using the wrinkling critical stress values, a wrinkling risk factor (perpendicular to the 1-direction) can be defined as

$$
f_{\sigma}=\frac{\sigma_{1}}{\sigma_{1}^{c r}}
$$

Therefore a wrinkling risk exists whenever $f_{\sigma}$ is larger than 1. Obviously, the risk is more important with larger values of the risk factors.

\subsection{Adaptive strategy}

In the adaptive procedure we need to detect the zones (elements) to be refined and determine a new size for those zones (elements). The zones detection is 
obtained by the use of the wrinkling risk factors (12). The new mesh size is obtained by the use of the wavelength of the wrinkles

$$
L_{1}=\frac{l}{\lambda_{1}}=\frac{\sqrt{R_{2} t}}{\lambda_{1}}
$$

to get a new weighted wrinkling mesh size as

$$
L^{w}=\frac{L_{1}}{m} \mathrm{f}_{\sigma}^{-1}
$$

with $m$ being the number of elements we wish to discretise a single wave with.

All of the above formulae have developed for wrinkling perpendicular to the 1direction. For wrinkling perpendicular to the 2-direction, a careful indices interchange is to be operated.

\subsection{General Algorithm for contact free wrinkling prediction}

Input the FE model and principal stresses (strains)

Loop over all elements

Depending on the signs of the principal stresses (strains), go to one of the four situations depicted in Figure 1. If Case I is encountered, go to next element. Else

Evaluate the principal curvatures

Evaluate the proportionality factor

Evaluate the critical stress (strain) and wavelength number for wrinkling perpendicular to the 1-direction (Case II)

Evaluate the critical stress (strain) and wavelength number for wrinkling perpendicular to the 2-direction (Case III)

Evaluate the critical stresses (strains) in both directions for Case IV and determine the predominant direction for wrinkling. Once the wrinkling direction is found evaluate the corresponding wavelength number

Evaluate the risk factor 
Go to next element
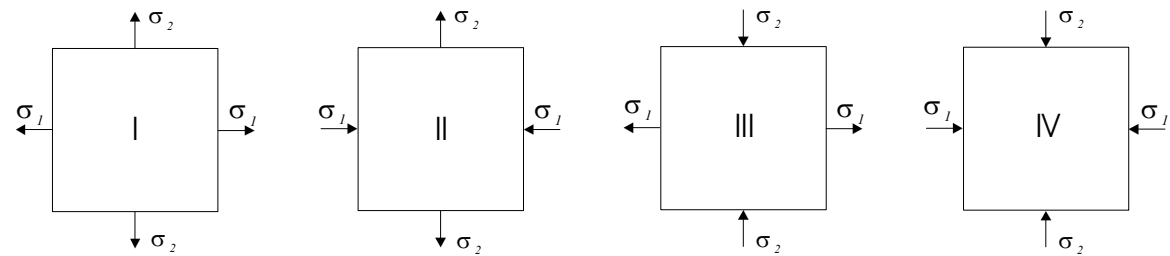

Figure 1. Possible Wrinkling directions

\section{Wrinkling with contact}

As already stated above, Hutchinson analysis is limited to contact free wrinkling. Therefore, in the contact zones a different approach had to be used. First, we considered Per Nordlund's [NOR 97] wrinkling indicator. Although, this indicator performs well in situations were strong rotations take place under strong compressive stresses, it did not, however, show the expect results when a product undergoes shallow wrinkling (Details will be given in a forthcoming publication). Therefore, a different indicator had to be used.

\subsection{A new wrinkling indicator}

The present indicator is based on the change of curvatures (during a single deep drawing step) under compressive stresses. This filters out all changes in curvature that are not due to compressive stresses, such as those caused for example by the geometry of the tool and the die. Such changes are, nevertheless, taken into account by the (geometric) error estimation indicator, if and when necessary.

The new wrinkling indicator in the ${ }^{i}$-principal direction is therefore defined as

$$
e_{i}^{w}=\frac{1}{A} \int\left|\frac{R_{i}^{t}-R_{i}^{t+\Delta t}}{R_{i}^{t} R_{i}^{t+\Delta t}}\right| d A \quad i=1,2
$$

with $i$ representing the principal curvature (stress) 2 direction and $R_{i}^{t}, R_{t}^{t+\Delta t}$ are the radii of curvatures in the $i$-direction at the beginning and at the end of a given deep drawing step, respectively.

Note that we do consider the principal directions (stress and curvature) one at a time as is the case with Hutchinson approach, and take the maximum change for 
each element. Consequently, the wrinkling indicator value $e^{w}$ is defined as the maximum of $e_{i}^{w}$, i.e.

$$
e^{w}=\max \left(e_{i}^{w}\right) \quad i=1,2
$$

Finally, we note that the present indicator can be viewed as a generalisation of the discretisation errors indicators in which a (relative) change, e.g. in the thickness or geometry, is measured between the finite element solution and a higher order solution obtained by some solution recovery technique. The change in the solution for the wrinkling indicator being between the solution at the beginning and the end of a given deep drawing step, considering only elements under compressive stresses in the 1 and/or 2 principal directions.

As for Hutchinson approach, the present indicator is local and is of a postprocessing type, and is therefore determined at a very low computational cost.

\subsection{Adaptive strategy}

Again, in the adaptive procedure we need to detect the zones (elements) to be refined and determine a new mesh size for those zones (elements). The zones detection is obtained by the use of the wrinkling indicator (16) and the new mesh size is obtained by the use of the following formula

$$
L^{w}=L \frac{e^{a v r}}{e^{w}}
$$

where $e^{a v r}$ is the average value of $e^{w}$ over all rotating elements and $L$ the old element size.

Moreover, to avoid excessive refinement, a check against a user specified minimum size is to be operated before the refinement actually takes place.

\subsection{General Algorithm for wrinkling prediction}

At a given step $\mathrm{N}$

Loop over all elements

Input all necessary data at the beginning and at the end of step $\mathrm{N}$

Evaluate the principal curvatures and their direction at the end of step $\mathrm{N}$ 
Determine the principal stress directions at the end of step $\mathrm{N}$

Evaluate the curvatures at the beginning of step $\mathrm{N}$

Compute the change in the curvature and the wrinkling indicator

Determine the new mesh size

Go to next element

\section{Numerical examples}

The performance of the wrinkling prediction procedure with adaptive mesh refinement described in this work is here demonstrated.

A hemispherical product is considered for wrinkling prediction analysis. The punch has a radius of $146.5 \mathrm{~mm}$ and the die shoulder a radius of $30 \mathrm{~mm}$. The initial sheet thickness is $1 \mathrm{~mm}$ and the product depth $100 \mathrm{~mm}$. Hollomon's hardening law is used with the following set of parameters : $K=542, n=0.228$ and $r=2.2$. In all numerical examples, we have used our implicit sheet metal forming code DiekA [DIE 00] with DST finite elements [BAT 89].

In the next subsections both wrinkling indicators along with the discretisation errors estimation are considered in situations where a high $(100 \mathrm{kN})$ as well as a low $(0.25 \mathrm{kN})$ blank holder force are used.

\subsection{Hemispherical product using the error estimator and the Hutchinson approach based wrinkling indicator}

In this numerical simulation a high blank holder force is used to avoid wrinkling under the blank holder as Hutchinson approach handles contact free wrinkling only.

Figure 2 shows the series of refinements obtained with a step size of $2 \mathrm{~mm}$. The simulation is started with a relatively coarse mesh comprising 2050 elements and ended with an adapted mesh of 5350 elements. The first refinement that takes place in step 15 is due to the thickness variation at the bottom of the product, the geometric error estimation in the region of the die shoulder and the wrinkling indicator that senses a potential for wrinkling in the body of the product and, mostly in anticipation, refines the mesh. In step 33, due to the mounting pressure under the blank holder in the die shoulder zone and because of the near flatness of the sheet the risk factor goes beyond one and consequently the refinement is intensified. In the final step, this effect is increased which brings the final mesh to 5350 finite elements. It is noted that six complete waves do develop in the body of the product. It should be noted that, when a coarse mesh is used, only four waves do develop. As 
will be seen in a forthcoming publication devoted to the comparison of numerical and experimental results, the coarse mesh does not produce the correct results.

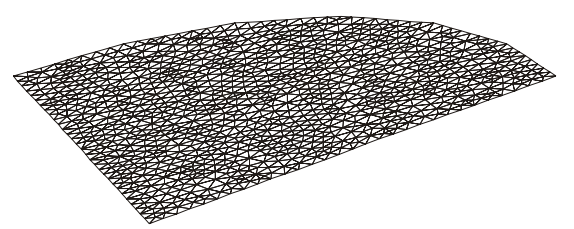

Initial Mesh - 2050 Elts

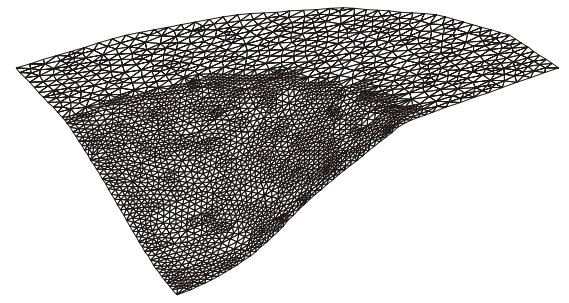

Step 33 - 4850 Elts

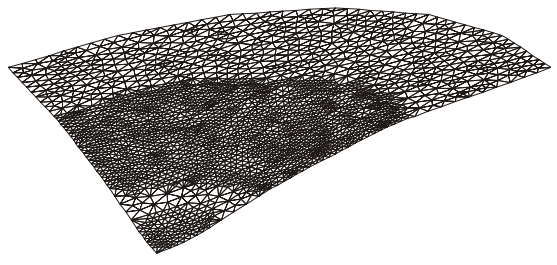

Step 15 - 4600 Elts

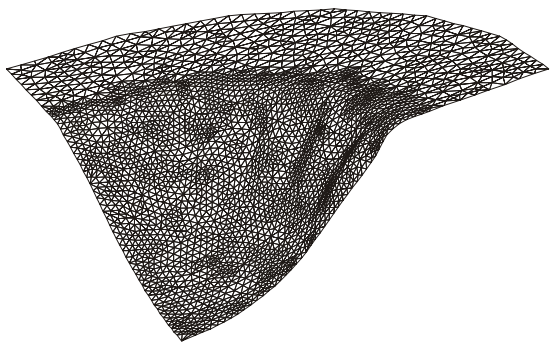

Step 49 - 5350 Elts

Figure 2. Mesh refinement for the hemispherical product using the error estimator and the Hutchinson approach based wrinkling indicator.

\subsection{Hemispherical product using the error estimator and the geometric wrinkling} indicator

A high and a low blank holder force are used in this example to distinguish between wrinkling with contact under the blank holder and the contact free wrinkling that takes place in the body of the product.

\subsubsection{Use of high blank holder force}

Figure 3 shows the obtained adapted meshes at deep drawing steps 17, 34 and 50. The simulation is started with an initial mesh of 2050 elements. A punch displacement of $2 \mathrm{~mm}$ per increment is used. The first refinement shown in Figure 3 at step 17 is due, as in the previous example, to the geometric error estimation (around the die radius), to the geometric wrinkling indicator (in body of the product) and to the thickness error estimation (at the bottom of the product). At the $34^{\text {th }}$ deep drawing step, as expected, the refinement is intensified as the geometry keeps changing around the die radius, the buckles continue their development and a higher jump in the thickness distribution is encountered at the bottom of the product. In step 50 some extra refinement is performed in the die shoulder zone. As can be seen 
in this example, the geometric wrinkling indicator behaves very much like Hutchinson approach based wrinkling indicator.

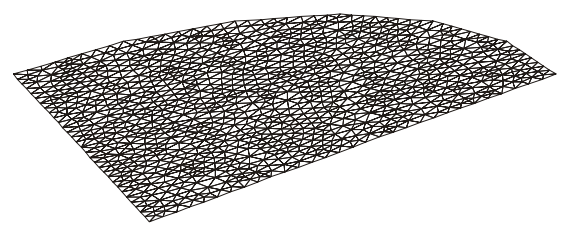

Initial Mesh - 2050 Elts

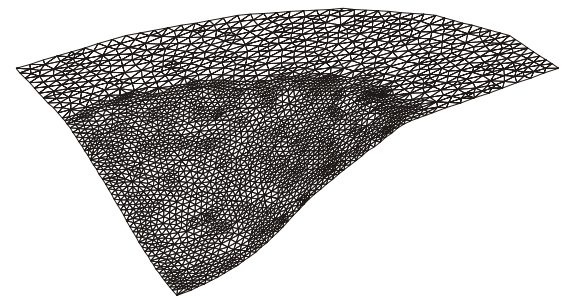

Step 34 - 4900 Elts

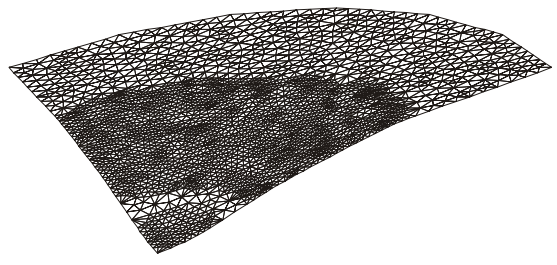

Step 17 - 4450 Elts

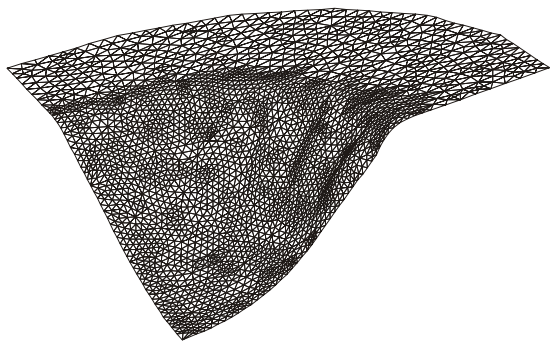

Step 50 - 5400 Elts

Figure 3. Mesh refinement for the hemispherical product using the error estimator and the geometric wrinkling indicator (Use of high blank holder force)

\subsubsection{Use of low blank holder force}

Figure 4 shows the obtained adapted meshes at deep drawing steps 32, 64 and 96. The initial mesh, again, comprises 2050 elements. However, a deep drawing step of $1 \mathrm{~mm}$ is used this time for stability reasons.

The first refinement shown in Figure 4 at step 32 is, as for the previous examples, due to the geometric error estimation (around the die radius), to the geometric wrinkling indicator (in body of the product) and to the thickness variation (at the bottom of the product). In step 64, the refinement is intensified around these regions. It is noted that the drawing behaviour so far and consequently the mesh refinement is similar to the previous case whereby a high blank holder force is used. In the final mesh at step 96, as expected, the refinement covers the whole area under the blank holder to properly describe the new buckles that develop in that region of the sheet. 


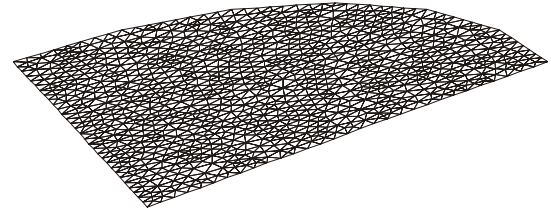

Initial Mesh - 2050 Elts

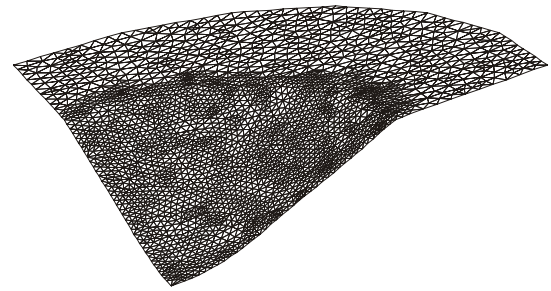

Step 64 - 5000 Elts

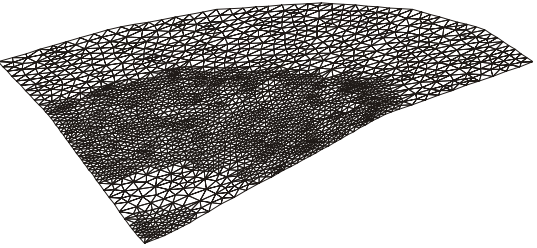

Step 32 - 4530 Elts

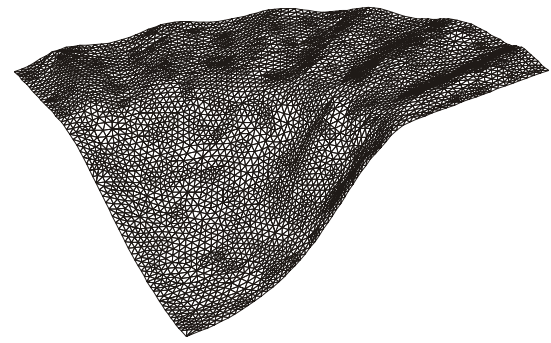

Step 96 - 8150 Elts

Figure 4. Mesh refinement for the hemispherical product using the error estimator and the geometric wrinkling indicator (Use of low blank holder force).

\section{Conclusions}

It has been demonstrated that the use of adaptive mesh refinement in wrinkling prediction analysis is a necessary approach for reducing the computational cost and better describing the wrinkling phenomena. However, it is also found that this has to be linked with an error estimation routine to properly approximate the curvatures and the thickness as these play a major role in the wrinkling process.

Hutchinson approach based wrinkling indicator that handles contact free wrinkling has been complemented with a new wrinkling indicator based on local curvature changes.

As a benchmark test, the hemispherical product has been considered with a high and a low blank holder force to distinguish between contact free wrinkling that takes place in the body of the product and wrinkling that takes place under the blank holder.

Given that the numerical simulation of complex sheet metal forming involve large scale models that necessitate a large to a very large number of finite element to discretise the products, the use of error estimation to better describe the curvatures and thickness distribution on one hand, and the use of wrinkling indicators on the 
other hand is becoming more of a must than an option to be taken especially that these indicators are local and are therefore determined at a very low cost.

\section{Acknowledgments}

This research was carried out under project number ME97033 in the framework of the Strategic Research programme of the Netherlands Institute for Metals Research in the Netherlands (www.nimr.nl).

\section{References}

[AMZ 91] AMEZIANE-HASSANI, H. and NEALE, K., On the analysis of sheet metal wrinkling, Int. J. Mech. Sci., 33, p. 13-30, 1991.

[BAT 89] BATOZ, J. L. and LARDEUR, P., A discrete shear triangular element with nine d.o.f for the analysis of thick to very thin plates, Int. J. Num. Meth.Eng., 28, p. $533-560,1989$.

[BON 92] BONET, J. and WOOD, R. D., Mesh enrichment procedures for the finite element analysis of thin sheet forming processes, Num. Meth. In Ind. Processes, Chenot, Wood and Zienkiewicz (eds.), Balkema, Rotterdam, p. 221 - 227, 1992.

[BON 94] BONET, J., Error estimators and enrichment procedures for finite element analysis of thin sheet large deformation processes, Int. J. Num. Meth. Eng., 37, p. 1573 - 1591, 1994.

[BRU 97] BRUNET, S., BATOZ, J. L. and BOUABDALLAH, S., Sur l'evaluation des risques de plissement locale de pieces industrielles obtenues par emboutissage, Actes $d u$ 3eme Colloque National en Calcul des Structures, p. 753-758, Giens, France, 1997.

[DIE 00] DiekA 6.1 Manual, University of Twente, 2000.

[GEL 98] GELIN, J. C., PASQUIER P. and BOUDEAU, N., Prediction of necking and wrinkling in sheet metal forming from the analysis of local equilibrium conditions, Numiform '98, J. Huetink and F.P.T. Baaijens (Eds.), Balkema, Rotterdam, 1998.

[HIL 58] HILL, R., A general theory of uniqueness and stability in elastic-plastic solids, J. Mech. Phys. Solids, 6, p. 236-249, 1958.

[HUT 74] HUTCHINSON, J. W., Plastic buckling, Adv. Appl. Mech., 14, p. 67-144, 1974.

[HUT 85] HUTCHINSON, J. W. and NEALE, K. W., Wrinkling of curved thin sheet metal, Plastic Instability, J. Salencon (Ed.), Press Ponts et Chaussees, p. 71-78, 1985.

[NAG 72] NAGHDI, P. M., The theory of shells and plates, Mechanics of Solids II, Via/2, Flugge S. and Truesdell C. (Eds.), Springer, 1972. 
[NEA 89] NEALE, K.W., Numerical analysis of sheet metal wrinkling, Numiform '89, Thompson et al (Eds.), Balkema, Rotterdam, p. 501-505, 1989.

[NEA 90] NEALE, K.W. and TUGCU, P., A numerical analysis of wrinkling formation tendencies in sheet metals, Int. J. Num. Meth. Eng., 30, p. 1595-1608, 1990.

[NOR 97] NORDLUND, P. and HAGGBLAD, B., Prediction of wrinkle tendencies in explicit sheet metal forming simulations, Int. J. Num. Meth. Eng., 40, p. 4079-4095, 1997.

[NOR 98] NORDLUND, P., Adaptivity and wrinkle indication in sheet metal forming, Comput. Methods Appl. Mech. Engrg. 161, p. 127-143, 1998.

[ROD 97] RODRIGUEZ-VILLA, A., Etude theorique et experimentale de l'extrusionsouflage de corps creux en polymere, PhD thesis, Ecole Nationale Superieure des Mines de Paris, France, 1997.

[SEL 90] SELMAN, A., HINTON, E. and ATAMAZ-SIBAI,W., Edge effects in MindlinReissner plates using adaptive mesh refinement, Eng. Comput., 7, p. 217-226, 1990.

[SEL 93] SELMAN, A., and HINTON, E., Two dimensional transient dynamic analysis with adaptive mesh refinement, Comm. Num. Meth. Eng., 9, p. 825-836, 1993.

[SEL 97] SELMAN, A., HINTON, E. and BICANIC, N., Adaptive mesh refinement for localised phenomena, Comp. \& Struct., 63, p. 475 - 495, 1997.

[SEL 00] SELMAN, A., MEINDERS, T., van den BOOGAARD, A.H. and HUETINK, J., Wrinkling prediction with adaptive mesh refinement, 3rd ESAFORM Conference on Material Forming, Stuttgart, 11-14 April, 2000.

[SEL 02] SELMAN, A., MEINDERS, T., van den BOOGAARD, A.H. and HUETINK, J., On

Adaptive Mesh Refinement in Wrinkling Prediction Analysis, $5^{\text {th }}$ ESAFORM Conference on Material Forming, Krakow, 14-17 April, 2002.

A. Selman holds a Master degree from the University of Wales - Swansea. He was privileged to be in the last MSc class taught by Prof. O.C. Zienkiewicz at Swansea. He also holds a $P h D$ degree from the same university and was honoured to be supervised by late Prof. E. Hinton.

He first joined the group of Prof. J.L. Batoz at Compiegne (France) in 1993, where he worked, amongst other things, on adaptive mesh refinement for $2 D$ and $3 D$ problems and on the stability analysis of metal cans.

In 1999 he joined the Netherlands Institute for Metals Forming with an office at the University of Twente where he works in the group of Prof. Huétink.

His research interests have been focused on adaptive mesh refinement in static and dynamic, linear and non-linear for $2 D$ and $3 D$ structural problems and more recently on wrinkling prediction analysis. 
T. Meinders holds a Master of Science degree in Mechanical Engineering from the University of Twente, the Netherlands.He also holds a PhD degree supervised by Prof. Huetink from the same university. Recently, he has been appointed as a staff member in the Applied Mechanics group of the University of Twente under the leadership of Prof. Huétink. His research interests have been focused on sheet metal forming, and in particular on tailored blanks, drawbeads, material models, adaptive mesh refinement and in improving the robustness of implicit codes.

A.H. van den Boogaard graduated in mechanical engineering at the University of Twente in 1988. From 1989 to 1995 he worked at the Netherlands Organisation for Applied Scientific Research (TNO) in the Computational Mechanics section of the Institute for Building and Construction Research where he took part in the development of the Diana finite element code. In 1995 he returned to the University of Twente to become a researcher/lecturer in finite element analysis. His current research focus is on sheet metal simulations and numerical methods.

J. Huétink studied Mechanical Engineering at the universities of Delft and Twente. He received his PhD degree from the University of Twente under the supervision of Prof. A. Rijken and Prof. J.F. Besseling. He worked at the Institute for Mechanical Engineering of the Dutch Organization for applied scientific research, TNO (1975-1980). He returned to the University of Twente, where in 1992 he was appointed as a full professor in mechanics of forming processes. He is member of the Program Council and cluster leader of the Netherlands Institute for Metals Research (NIMR). He is member of the NUMIFORM Steering Committee and organizer of NUMIFORM '98 conference and member of the Board of Directors ESAFORM (European Scientific Association for Material Forming). 